\title{
Características geológico-geotécnicas y propuestas para la preservación del Sitio y Monumento Histórico n³8, Cabaña de Nordenskjöld, Antártida
}

\author{
Adrián Silva Busso ${ }^{(1,2)}$ y Yevgueniy Yermolin ${ }^{(3)}$ \\ (1) Universidad Buenos Aires, Facultad de Ciencias Exactas y Naturales, Departamento de Geología, \\ Ciudad Universitaria Pabellón II, 1 piso, of.1, Ciudad de Buenos Aires, Argentina (1428). \\ silvabusso@yahoo.com.ar \\ (2) niversidad Tecnológica Nacional, Facultad Regional Concordia, Dpto. de Ingeniería Civil, GIICMA, \\ Salta 277, Concordia, Entre Ríos, Argentina (3200). \\ (3) Instituto Antártico Argentino, Depto.de Ciencias de laTierra, Cerrito 1248, Ciudad de Buenos Aires (1010). \\ ivgen52@yahoo.com

\begin{abstract}
RESUMEN
El Sitio y Monumento Históricon³8 (Cabaña de Nordenskjöld) está situado en la Isla Cerro Nevado, archipiélago James Ross, Antártida. Constituye un sitio de valor patrimonial e histórico importante, ya que existen en la Antártida pocas construcciones centenarias. La cabaña fue construida en Febrero de 1902 para servir de refugio a la expedición sueca dirigida por Otto Nordenskjöld, realizada entre 1901 y 1904 . Su ubicación, en la coronación de un terraplén con permafrost rico en hielo determina las condiciones inestables de cimentación que se han manifestado intensamente en las últimas décadas, principalmente como consecuencia del aumento de temperatura. En el entorno se observan procesos de erosión relacionados con el aumento de descongelamiento estacional y fusión del hielo subterráneo en el techo del permafrost. También factores antrópicos han acelerado el proceso, por ejemplo en el pasado el uso de la cabaña como refugio y garaje durante las campañas de verano. El terraplén contiene hielo subterráneo que puede alcanzar del 17 al $20 \%$ yel espesor de la capa activa tiene una media de $1.2 \mathrm{~m}$. Se ha estimado un asentamiento potencial máximo del replano sobre el terraplén de entre 0.15 y $0.25 \mathrm{~m}$, producto de la fusión en el techo del permafrost, lo que podría modificar la superficie natural y llegar a producir daños significativos. Este estudio analiza estrategias de contingencia y mitigaciónpara evitar o reducir la tasa de asentamiento y evitar alterar la coronación del terraplén. El objetivo es proteger los espaldones y cimientos de la erosión superficial y la termoerosión. Para ello se propone aumentar el espesor del terraplén, realizar un armazón sobre el espaldón del terraplén e instalar un zócalo o barrera de contención en los cimientos.
\end{abstract}

Palabreas clave: Cabaña de Nordenskjöld, Antártida, Isla Cerro Nevado, Sitio Histórico, Permafrost

\section{Geologic - geotechnical characteristics and preservation proposals for the

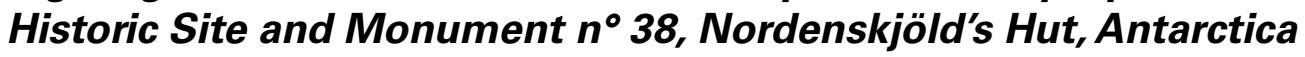

\begin{abstract}
The historic site and monument $n^{\circ} 38$, Nordenskjöld Hut, is located on Snow Hill Island, James Ross archipelago, Antarctica. This is a place of a great historic and heritage value because there are very few centennial buildings in Antarctica. This building was built in February 1902 as a refuge by the Swedish expedition directed by Otto Nordenskjöld, between 1901 and1904. The building is located over an embankment with ice-rich permafrost, which produces unstable conditions for the foundation. In the past anthropic factors accelerated this process, for example the use of the hut as refuge and garage for other field campaigns. Intensive and potentially destructive signs are observed as a consequence of the thermokarst and erosion active processes in the last decades, in relation with climate warming. Around the embankment the erosion processes are
\end{abstract}


Silva Busso, A. y Yermolin, Y., 2017. Características geológico-geotécnicas y... Boletín Geológico y Minero, 128 (1): $207-220$

connected with underground icethaw occurring in the top of the permafrost. The embankment area contains $17-20 \%$ of underground ice and the mean active layer depth is $1.2 \mathrm{~m}$. A potential maximum settlement of the embankment top surface between $0.15-0.25 \mathrm{~m}$ has been estimated. The above mentioned processes are consequence of ice thaw in the permafrost table. This would modify the natural surface and could produce significant damages. This study analyzes contingency and mitigation strategies to prevent or reduce the rate of settlement and avoid altering the top of the embankment. The goal is to protect the slopes and foundation from surface erosion and thermo-erosion. We propose increasing the thickness of the embankment, making a frame on the shoulder of the embankment and carrying out a retaining wall or socket in the foundation.

Keywords: Nordenskjöld Hut, Antarctica, Snow Hill Island, historic site and monument, permafrost

\section{ABRIDGED ENGLISH VERSION}

\section{Introduction}

The historic site and monument (HSM) $n^{\circ} 38$ is located on Snow Hill Island, James Ross archipelago, Antarctica. This is a place of a great historic and heritage value because there are very few centennial buildings in Antarctica (Fig. 1). This building was built in 1902 as a refuge by the 1901-1902 Swedish expedition directed by Otto Nordenskjöld. After seventy years with no maintenance the Argentina Antarctic Institute started restoration in the 1979-1980 campaign (Comerci, 1983). Its location, on the top of a natural embankment with ice-rich permafrost, determines the unstable foundation conditions that have been evident in recent decades, mainly due to the temperature increasing during the austral summer. This paper aims to study the current state and propose possible solutions to improve the stability of the embankment using local materials and geotechnical measures, and analyzes contingency and mitigation strategies to prevent or reduce the rate of settlement and avoid altering the top of the embankment (Fig. 2).

\section{Methods}

An analysis of the characteristics and evolution of the permafrost active layer was carried out. Meteorological data of the period 1971-2010 from Marambio Base (Seymour/Marambio Island, $22 \mathrm{~km}$ from Snow Hill Island) were used. Vertical electrical soundings (VES) were carried out in the HSM $n^{\circ} 38$ area with the purpose of knowing the depths and ice or water content. The involved geotechnical and geocriological processes were inventoried and soil samples in and around the embankment were obtained. Pits in the active layer and thermal sounding were used to obtain subsurface samples and measurements. Laboratory analysis included soil grain size, total moisture, density and thermal proprieties. These data allowed the calculation of the maximum summer thawing thickness (Ermolin and Skvarca, 2004) and how the land settlement may affect the top of the embankment. For the soil study the HRB classification was used to identify the permafrost zones based on the United Soil Classification System, Corps of Engineers and Bureau of Reclamation (Linell and Jonston, 1973). This soil classification depends on the thawing reaction (F) shown in Table 1.

\section{Results}

The hut is located in the upper part of an embankment with ice-rich permafrost that produces unstable conditions for the foundation. Active and potentially destructive processes, such as thermokarst and erosion, have been observed in the last decades, in connection with increasing temperatures. The most serious threat for the HSM $n^{\circ} 38$ is the slope instability of the embankment due to the soil ice content changes. The active layer is composed of a mixture of poor sorted unconsolidated sediments exposed to runoff in summer. Six sectors have been differentiated in the HSM $n^{\circ} 38$ embankment that could produce serious modifications in the topography in the future (Figs. 3, 4 and 5). Figure 6 shows the study area, including land forms in the $H S M n^{\circ} 38$. The hut is located on the bottom of a moraine of the Snow Hill Glacier. Figure 6 shows the upper embankment surface with ice-rich permafrost (1), the embankment slope (2), the construction aggregates around the hut (3), the metal and wood pallet defense (4), the construction aggregate in the embankment slope (5), and the geocriologic profile location $\left(A-A^{\prime}\right)$. Erosion processes occur connected with increased permafrost thaw in the permafrost table.

In situ and laboratory measurements of soil samples of the active layer show us that the ice content varies between $17-20 \%$. However, some soil samples contain up to $30 \%$ of interstitial ice. The active layer 
Silva Busso, A. y Yermolin, Y., 2017. Características geológico-geotécnicas y... Boletín Geológico y Minero, 128 (1): $207-220$

depth in the embankment top is $1.2 \mathrm{~m}$ in the south part, $1.0 \mathrm{~min}$ on the east side and $1.4 \mathrm{~m}$ on the west and north sides. The day positive-degree rate was calculated to determinate the summer thawing thickness. The temperature data (Fig. 7) allowed us to calculate the day positive-degree rate. The results are rate values of day positive-degree between 300 y $600^{\circ} \mathrm{C} /$ day. Vertical electrical soundings (VES) carried out on the embankment top in HSM $n^{\circ} 38$ (Fig. 8) allowed us to identify different resistive layers that affect the local geocriology and hydrogeology. The first layer is $1.30 \mathrm{~m}$ deep and shows $30.9 \mathrm{\Omega m}$ resistivity. We can interpret it as a mixture of sand, silt and freshwater corresponding to the active layer. The second layer is $5.50 \mathrm{~m}$ thick, up to $6.8 \mathrm{~m}$ deep and $1960 \mathrm{\Omega m}$ resistivity. This resistivity increment is interpreted as more ground ice in the thawing zone, contained in a mixture of fine sand and silt. Finally, the third layer has $176 \Omega \mathrm{m}$ resistivity and an unknown thickness due to the small size of the surface on the embankment that limited the VES measurements. This layer is interpreted as containing dry permafrost (5-7\% ice) in silt and clay, typical on the Snow Hill and Marambio/Seymour islands.

\section{Discussion and conclusions}

Environmental conditions affect slope stability in permafrost areas, with the most important factors being the summer temperature regimen, supra-permafrost groundwater presence, active layer thickness, thawing changes, and soils characteristics.The runoff in the embankment may induce a uniform laminar erosion upon the surface due to the flow movement digging channels no more than $0.30 \mathrm{~m}$ deep. The supra-permafrost groundwater supply from the summer aquifer is related to the active-layer ice thaw and the summer precipitation. Water flow in the creeks only occurs in summer. It depends on the clastic rocks porosity and the local meteorological conditions.

Field and laboratory data have been used to calculate the local thickness of the thawing layer with the Nelson equation, deduced by the general Stefan equation (Nelson et al., 1997). These authors calculated the local thickness of the thawing layer. Using these data a potential maximum subsidence on the embankment top has been estimated between 0.15 and $0.25 \mathrm{~m}$. This potential subsidence must be considered as the highest value obtained in the warmest Antarctic summer scenario. With colder summers, or with more days with negative temperatures, the potential subsidence would be less. Figure 10 shows a schematic cross section of the embankment at the HSM $n^{\circ} 38$, showing sedimentological and permafrost related features. The existing situation is the consequence of ice thaw in the permafrost top, which has modified the natural surface and could produce significant damage. To sum up, the embankment contains $17-20 \%$ underground ice and a mean active layer thickness of $1.2 \mathrm{~m}$. The potential maximum settlement of the top surface of the embankment has been estimated between 0.15-0.25m.

\section{Introducción}

El Sitio y Monumento Histórico (SMH) n³8 “Cabaña de Nordenskjöld" está situado en la Isla Cerro Nevado, archipiélago James Ross, Antártida (Figura 1). Posee un considerable valor patrimonial e histórico, ya que en la Antártida existen pocas construcciones centenarias. La cabaña fue construida por la Expedición Sueca dirigida por el Dr. Otto Nordenskjöld, realizada entre 1901 y 1904.

Su ubicación, en la coronación de un terraplén natural con permafrost rico en hielo, determina las condiciones inestables de cimentación, que se han manifestado en las últimas décadas principalmente como consecuencia del aumento de la temperatura durante el verano austral. En los alrededoresde la cabaña se observan fenómenos de termokarst y erosión por aumento de descongelamiento estacional y fusión de los hielos subterráneos en el techo del permafrost. El mantenimiento de esta construcción ha sido un objetivo permanente del Instituto Antártico Argentino
(IAA) en los últimos 40 años. Este estudio tiene por objeto estudiar la situación existente y proponer posibles medidas geotécnicas tendentes a mejorar la estabilidad del terraplén utilizando materiales locales.

La Cabaña de Nordenskjöld fue construida en la Isla Cerro Nevado (aprox. $64^{\circ} 24^{\prime} \mathrm{S}, 57^{\circ} 40^{\prime} \mathrm{W}$ ), en febrero de 1902, por el grupo principal de la Expedición Sueca dirigida por el Dr. Otto Nordenskjöld. El refugio, fue habitado en aquella ocasión por las seis personas que invernaron, entre ellos el alférez argentino José María Sobral. La cabaña estuvo abandonada desde noviembre de 1903 (fecha en que la expedición fue rescatada por la corbeta Uruguay). Si bien la Armada Argentina realizó diversas tareas de reparación en diversas oportunidades a lo largo del siglo XX, las labores con fines específicos de restauración deledificio comenzaron en la campaña del verano austral 1979-1980 de la Dirección Nacional del Antártico (Comerci, 1983). Las primeras tareas de restauración fueron realizadas en el interior del refugio y su objetivo fue deshacer el hielo formado por la acumulación 


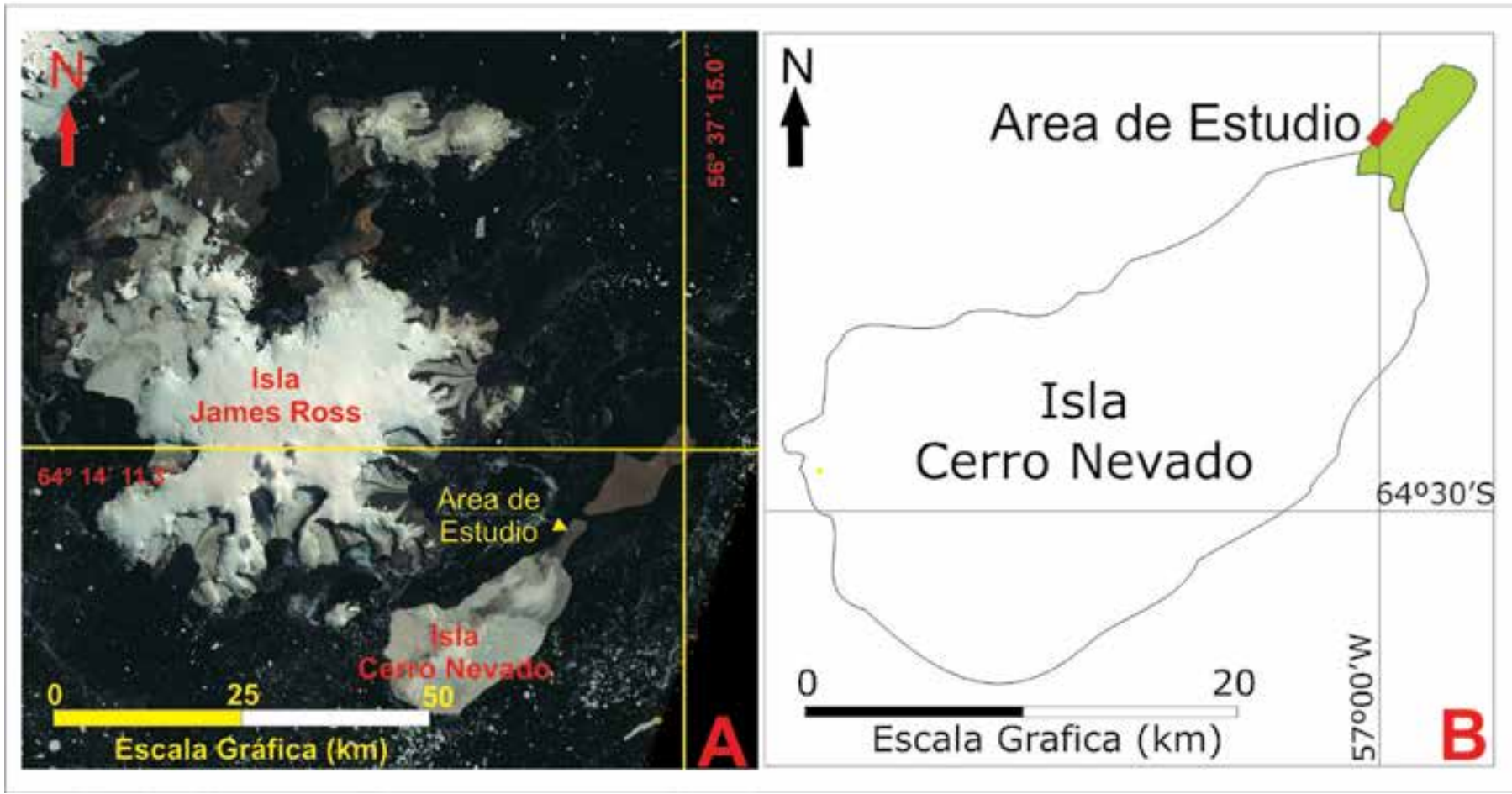

Figura 1. Situación de la Cabaña de Nordenskjöld en la Isla Cerro Nevado (A yB).

Figure 1. Location of Nordenskjöld's Hut on Snow Hill Island (A and B).

de nieve a consecuencia del deterioro de la estructura durante los casi 70 años quecareció de mantenimiento. La acumulación original, que alcanzaba una altura de $1.80 \mathrm{~m}$ y rellenaba todo el interior de la cabaña, fue descongelada y/o extraída tratando en lo posible de no dañar los componentes y objetos históricos de la expedición, que el mismo hielo había conservado durante ese tiempo (Capdevila, 1995).

En las siguientes etapas, se procedió a las tareas de reparación y mantenimiento de la parte externa de la construcción para la recuperación de los daños debidos al ambiente antártico. Se realizó el refuerzo de la pared norte, fuertemente deteriorada y con un alto riesgo de colapso, apuntalando la misma a modo de "contrafuerte", empleando tablas de madera que sostienen la pared a la altura del dintel norte de la cabaña (Figura 2). También se cubrió la estructura con pintura negra con la finalidad de aumentar el calor absorbido por la misma. A partir de la Campaña Antártica del Verano (CAV) 1993-94 empezaron los trabajos para el fortalecimiento del terraplén sobre el que asienta la construcción. Estas tareas no siempre han cumplido con los estándares mínimos de mantenimiento del patrimonio histórico y muchas de ellas han sido de carácter informal u ocasional.

Con el paso de los años, el terraplén había sufrido algunas modificaciones superficiales por acción de

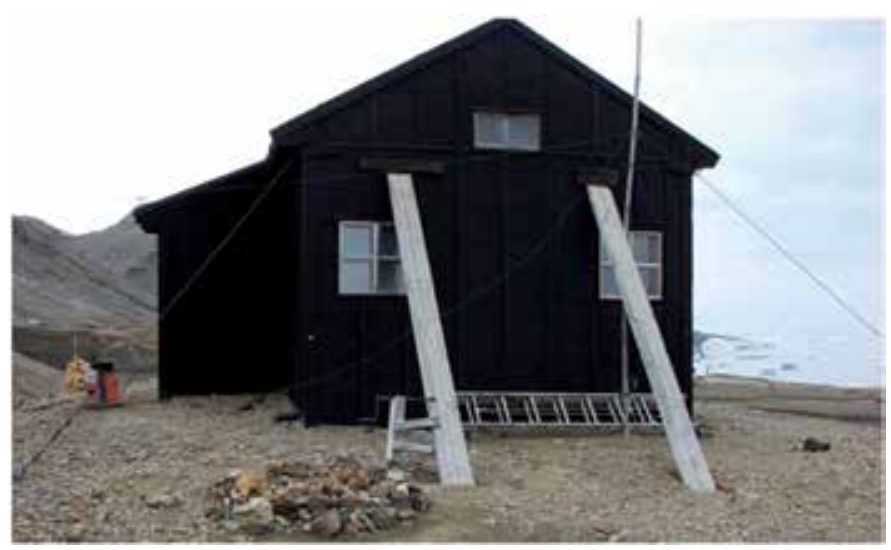

Figura 2. Reparaciones de la estructura externa y apuntalamiento del actual Museo Nordenskjöld.

Figure 2. External structure repair and proppingof the Nordenskjöld Museum.

la erosión y el asentamiento local de la superficie de coronación del mismo. Primeramente fue revestido de madera, fijado en distintos puntos con varillas de hierro, rodeando el terraplén con relleno desedimentos locales transportados desde otro sitio de la zona alejado de la cabaña. La urgencia de la reparación determinó la adopción de esta medida de contingencia 
provisional hasta realizar un estudio adecuado que redundase en soluciones a más largo plazo.

Con objeto de evaluar el estado actual de lacimentación de la estructura y proponer las tareas para mejorar la estabilidad dela misma fueron realizadas algunas determinaciones y estudios de las condiciones geocriológicas e hidrogeológicas durante la CAV2005/06 (en elmarco del proyecto PICTA 2005 “Permafrost y cambio climático en Antártica). Posteriormente, entre los años 2008 y 2012 el Programa de Gestión Ambiental y Turismo (de la Dirección Nacional del Antártico) ha llevado a cabo un proyecto para la puesta en valor del $\mathrm{SMH} \mathrm{n}^{\circ} 38$ (RCTA, 2003, 2010, 2011). Las primeras actividades de conservación preventiva de los materiales presentes se efectuaron en las CAV 2010/2011 y CAV 2011/12, específicamente en el sector del terraplén. Debe considerarse que las tareas de reparación del terraplén podrían conducir a afectar al registro arqueológico factor a considerar en cualquier propuesta de restauración.

\section{Materiales y métodos del estudio}

Se han tenido en cuenta los datos climáticos de la Base Marambio (situada a $22 \mathrm{~km}$,en la isla homónima y contigua a la de Cerro Nevado) del periodo 1971-2010 (Servicio Meteorológico Nacional) y empleándose en el análisis de las características y evolución de la capa activa y delpermafrost. Se ha realizado un sondeo eléctrico vertical (SEV) con el objeto de interpretar los contenidos de agua y hielo en el terraplén y su zona de influencia. Se trata de una metodología clásica usada en numeras ocasiones desde hace tiempo.Se realizó un inventario geocriogénico-geotécnico y se eligieron puntos de muestreo litológico en la zona del terraplén y área circundante. Se efectuaron calicatas en la capa activa y sondeos térmicos en distintos sectores del terraplén. Las tareas de laboratorio (LEGAN-IAA, Mendoza) han comprendido: granulometría, humedad total, densidad y propiedades térmicas de suelos clásticos típicos. Esta información es necesaria para calcular el espesor máximo de descongelamiento estacional (Ermolin y Skvarca, 2004). Este valor se ha usado para determinar el asentamiento potencial que podría afectar a la coronación del terraplén. En el presente trabajo se ha optado por utilizar la sistematización de suelos HRB y la clasificación de los suelos utilizada en las zonas de permafrost basada en United Soil Classification System, Corps of Engineers y Bureau of Reclamation (Linell and Jonston, 1973). La clasificación según su reacción al congelamiento (F) se puede observar en la Tabla 1.

\section{Análisis y Diagnóstico}

\section{Estado actual del terraplén y del asentamiento}

Uno de los problemas más graves que amenazan al $\mathrm{SMH} \mathrm{n}^{\circ} 38$ es la inestabilidad del terreno en la zona, con permafrost rico en hielo yuna capa activa compuesta por material clástico de acarreo, por lo tanto muy poco consolidado y sometido a la acción modificadora del cambio climático y el arrastre por agua superficial y suprapermafrost en épocas estivales. El $\mathrm{SMH} \mathrm{n}^{\circ} 38$ está ubicado sobre material morrénico de fondo del glaciar Cerro Nevado. En las proximidades existen otros depósitos de semejante origen señalados con flechas en la Figura 3.

\begin{tabular}{|l|l|c|l|}
\hline \multicolumn{1}{|c|}{ Clasificación } & \multicolumn{1}{|c|}{ Tamaño de partícula } & $\begin{array}{c}\text { \% de partículas <2 } \\
\mathbf{m m}\end{array}$ & \multicolumn{1}{c|}{ Tipos de suelos típicos } \\
\hline F 1 & Gravas & $3-10$ & GM, GP, GW-GM, GP-GM \\
\hline F 2 & Gravas arenosas & $\begin{array}{c}10-20 \text { y } \\
3-15\end{array}$ & $\begin{array}{l}\text { GM, GW-GM, GP-GM, SW, SP, SM, } \\
\text { SW -SM, SP-SM }\end{array}$ \\
\hline F 3 & Gravas arenosas sin Limo-Arena & $>20$ y $>15$ & $\mathrm{GM}, \mathrm{GC}, \mathrm{SM}, \mathrm{SC}$ \\
\hline F 4 & $\begin{array}{l}\text { Arcillas Limos en general Limos } \\
\text { arenosos }\end{array}$ & $\mathrm{CL}, \mathrm{CH}, \mathrm{ML}, \mathrm{MH}, \mathrm{SM}$ \\
\hline F 5 & $\begin{array}{l}\text { Arcillas, sedimentos de grano fino en } \\
\text { capas o bandas }\end{array}$ & & $\begin{array}{l}\mathrm{CL}, \mathrm{CL}-\mathrm{ML}, \mathrm{CL} \text { y } \mathrm{ML}, \mathrm{CL}, \mathrm{CH} \text { y } \mathrm{ML}, \mathrm{CL}, \\
\mathrm{CH}, \mathrm{ML} \text { y } \mathrm{SM}\end{array}$ \\
\hline
\end{tabular}

Tabla 1. Clasificación de suelos en zonas de permafrost (Linell and Jonston, 1973).G: Grava, S: Arena, C: Arcilla, P: Selección pobre, W: Selección buena, H: Alta, M: Media, L: Baja. 


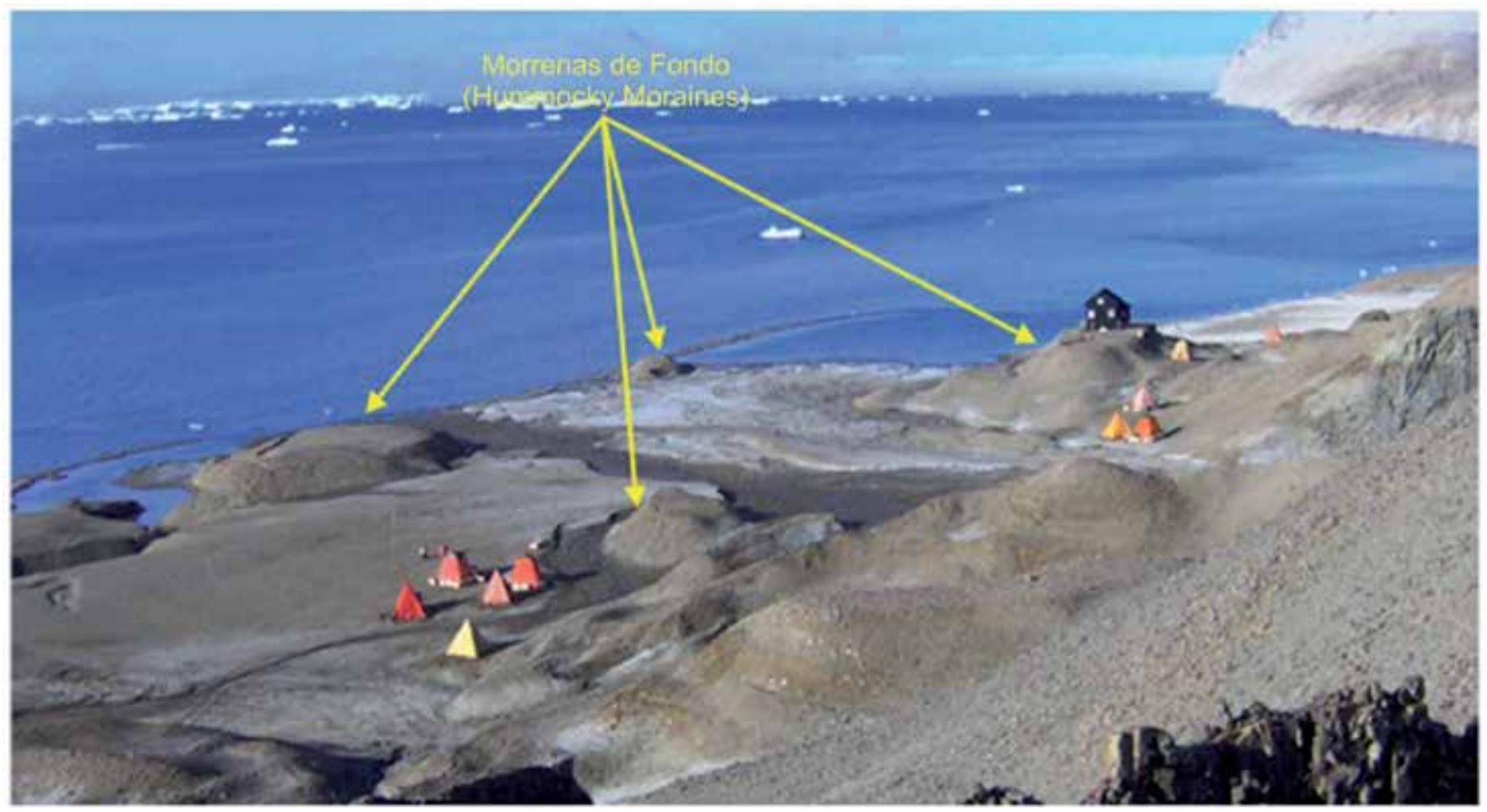

Figura 3. Morrenas del fondo con hielo intersticial. Sobre una de ellas se encuentra el actual monumento histórico.

Figure 3. Moraine deep sections with interstitial ice. On one of them is the historical monument.

Las tareas llevadas a cabo previamente (Capdevilla, 1995) lograron detener algunos procesos de erosión superficial y estabilizar parcialmente los márgenes, así como parte de la coronación y del talud del terraplén. Han sido tareas simples (fundamentalmente relleno de cárcavas, vías de escorrentía y nivelación con materiales locales) que atendieron la contingencia momentánea sin control posterior de la evolución del proceso. No obstante, actualmente se pueden identificar 9 sectores sobre el espaldón del terraplén que han sufrido desgaste por erosión y que podrían producir a lo largo del tiempo modificaciones graves del relieve original (Figuras 4 y 5 ).

Las condiciones morfológicas de los suelos de la capa activa en los sectores elegidos, incluida la coronación del terraplén, pueden resumirse en los siguientes términos:

- Coronación del Terraplén: Es un plano superior sub-horizontal con ligera inclinación al norte. Los márgenes de la estructura están rellenos con grava $y$ arena.

- Sector 1(Fig.4): Con superficie uniforme y pendiente orientada hacia el sur con $25-27^{\circ}$ de inclinación. En la parte inferior se encuentra una banda de rocas (artificial, sin función alguna).

- Sector 2 (Fig.4): Con superficie uniforme y pendiente orientada hacia el sur con $18-20^{\circ}$ de inclinación. En la parte inferior se encuentra una banda de rocas (artificial, sin función alguna).

- Sector 3 (Fig.4): Con superficie uniforme y pendiente orientada hacia el este con $20-22^{\circ}$ de inclinación. En la parte superior y parte inferior se encuentra una banda de rocas (artificial, sin función alguna).

- Sector 4 (Fig.4): Con superficie irregular y pendiente orientada hacia el este con $18-22^{\circ}$ de inclinación. En la parte superior se encuentra un murallón con paneles de aluminio, reforzados con piedras y en la parte inferior se encuentra una banda de rocas (artificial, sin función alguna).

- Sector 5 (Fig.5): Con superficie irregular y pendiente orientada hacia el norte con $22-24^{\circ}$ de inclinación.

- Sector 6 (Fig.5): Con superficie regular y pendiente orientada hacia el noroeste con $24-25^{\circ}$ de inclinación.

- Sector 7 (Fig.5): Con superficie irregular y pendiente orientada hacia el oeste con $22-23^{\circ}$ de inclinación. En la parte superior se encuentran dos pantallas construidas con paneles de aluminio (piezasportátiles de la vieja pista de aluminio de la Base Marambio), reforzadas con gravas locales en la parte inferior y en el centro. 
Silva Busso, A. yYermolin, Y., 2017. Características geológico-geotécnicas y... Boletín Geológico y Minero, 128 (1): $207-220$

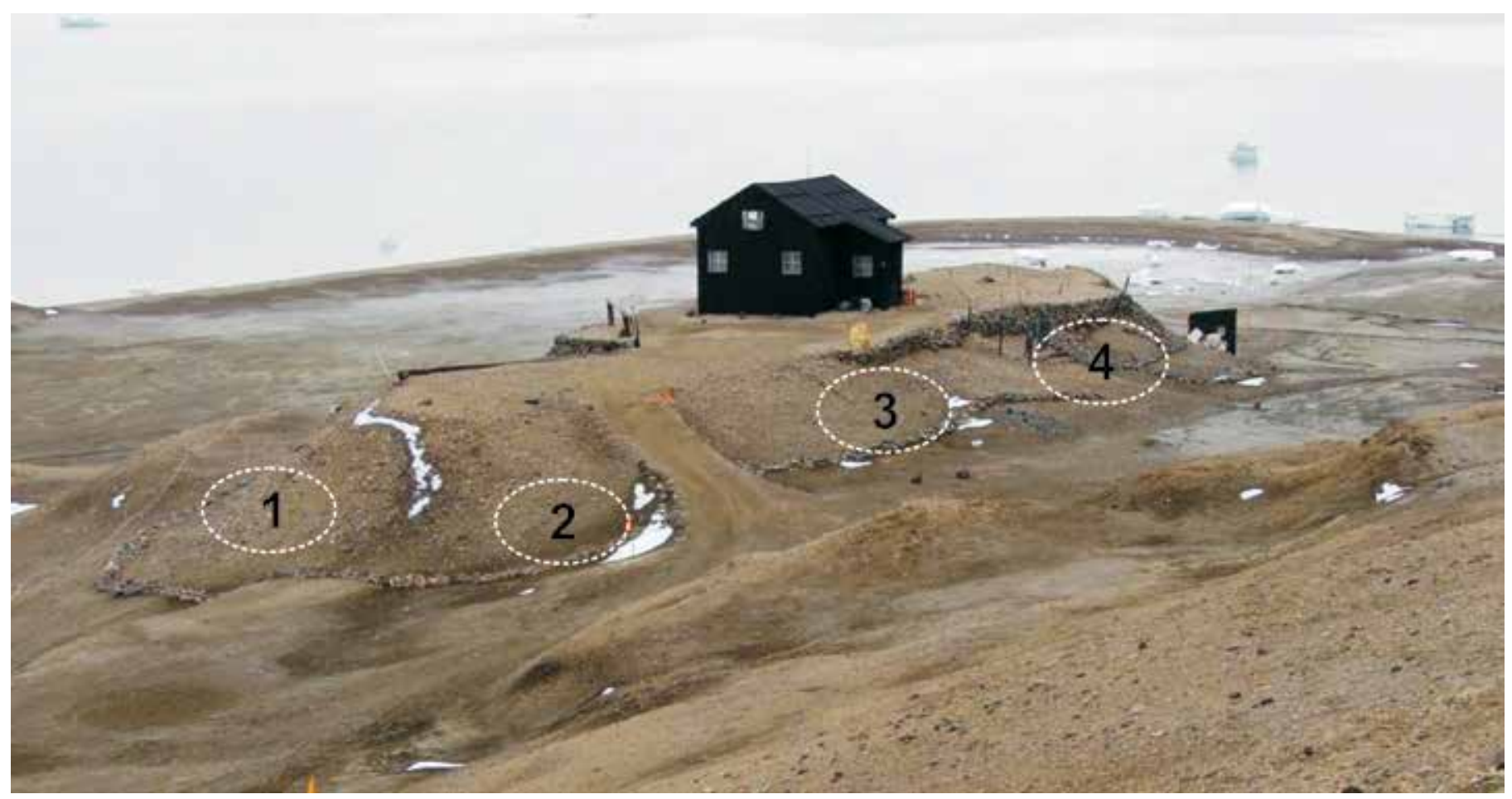

Figura 4. Sectores de muestreo en la parte sur del terraplén y este de la cabaña Figure 4. Sample sectors in the south part of the embankment and east of the hut.

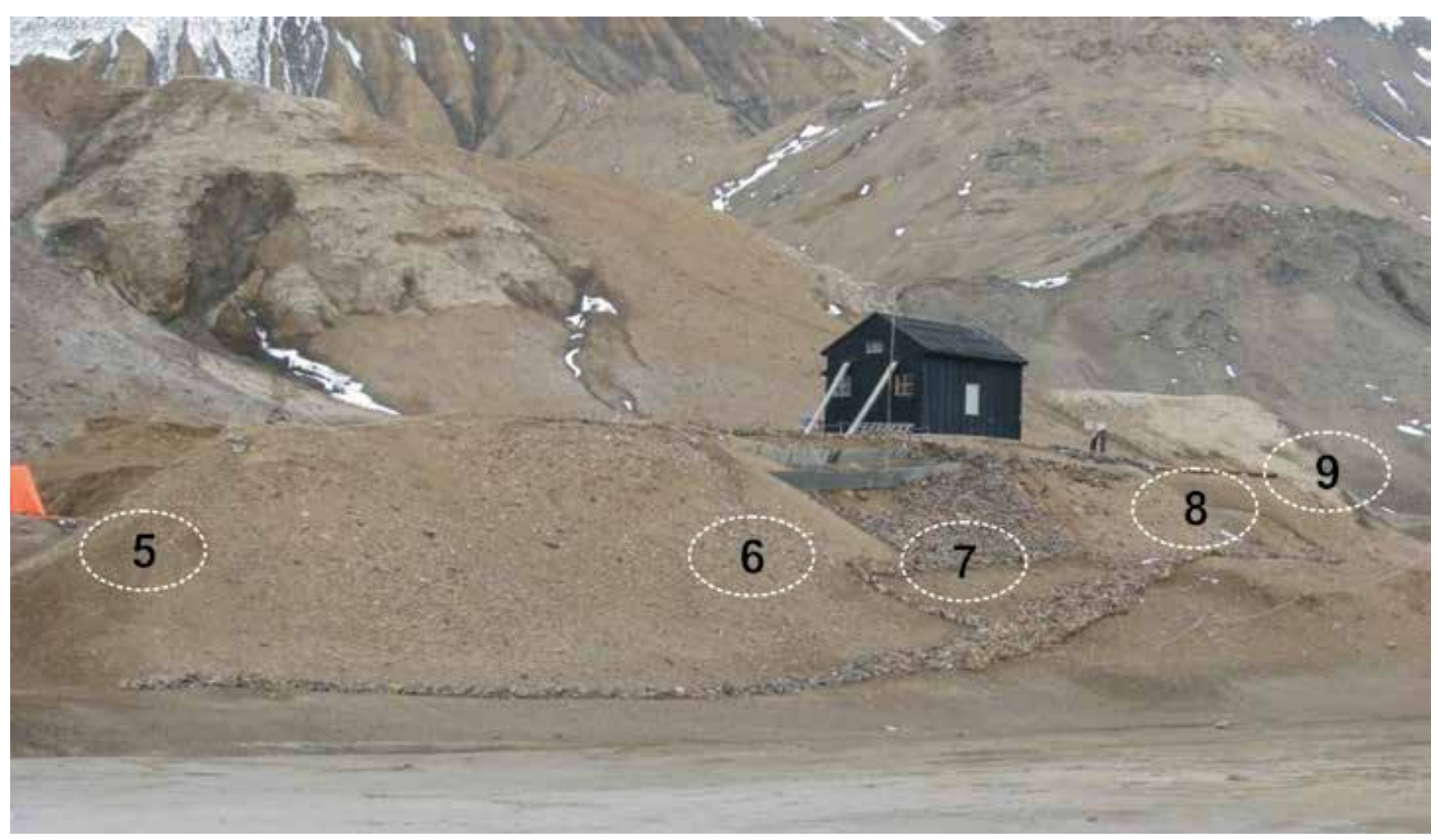

Figura 5. Sectores de muestreo en la parte norte del terraplén y oeste de la cabaña. Figure 5. Sample sectors in north part embankment and west of the hut. 
- Sector 8(Fig.5): Con superficie irregular y pendiente orientada hacia el oeste con $20-22^{\circ}$ de inclinación. En parte en parte superior (el margen de la superficie es plana) está reforzada con los antiguos troncos de palmera que sirvieron antes en el campo de antenas de la Base Marambio. En la parte inferior y en el centro se encuentra una banda de rocas (artificial, sin función alguna).

- Sector 9(Fig.5): Con superficie irregular y pendiente orientada hacia el noroeste con $24-25^{\circ}$ de inclinación, con la parte inferior reforzada con una banda de rocas (artificial, sin función alguna).

Las siglas de la Tabla 2 indican las características de la coronación y el talud según su estabilidad. Se considera estable cuando la capa activa no presenta profundización anómala ( $\mathrm{E}$ - terreno estable). Es moderadamente estable cuando se observan algunas evidencias de profundización de la capa activa (ME - terreno con moderada estabilidad).

Se considera inestable cuando la nieve acumulada por el viento podría fundirse y producir erosión en el terreno o el talud (IE - terreno inestable). Se ha podido detectar procesos de termokarst (TK), procesos o erosión (TE) y levantamiento por congelamiento estacional LC.

La figura 6 muestra la vista en planta del área de estudio circundante al $\mathrm{SMH} \mathrm{n}^{\circ} 38$ ubicado sobre una parte de la morrena de fondo del glaciar Cerro Nevado.

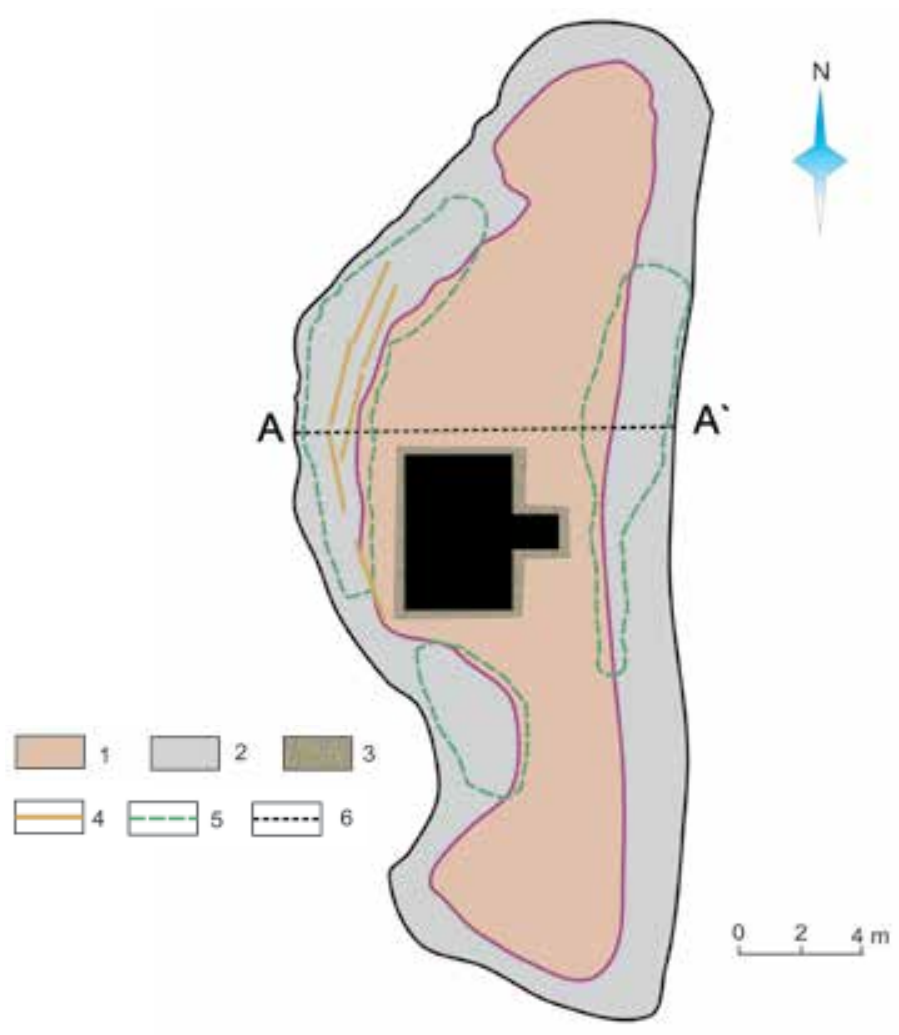

Figura 6. Plano del terraplén donde se sitúa la Cabaña de Nordenskjöld. Puede observarse: (1), el talud del terraplén (2), los márgenes del refugio rellenos con áridos (3), las defensas de contención hechas en madera y/o paneles de aluminio (4) y los márgenes del relleno en los taludes del terraplén (5). A- $A^{\prime}$ indica la localización del perfil mostrado en la figura 10.

Figure 6. Embankment map of Nordenskjöld's Hut. We can observe: (1) the slope of the embankment, (2) margins of the refuge stuffed with aggregates, (3) defense containment made of wood and/or aluminum panels, (4) and margins of the filling on the slopes of fill, (5). A-A 'indicates the location profile shown in Figure 10.

\begin{tabular}{|l|l|l|}
\hline \multicolumn{1}{|c|}{ Sector } & \multicolumn{1}{|c|}{ Características } & \multicolumn{1}{c|}{ Tipo de suelos } \\
\hline Superficie Terraplén & ME, TK. & F 3, GM \\
\hline Sector 1 & E, TE & F 2, GP y SM \\
\hline Sector 2 & E, TE & F 2, y SM \\
\hline Sector 3 & ME, TE & F 2, GM y SM \\
\hline Sector 4 & ME, TE, LC & F 2, GW-GM \\
\hline Sector 5 & ME, TE & F 2 y F 4, ML, GW-GM \\
\hline Sector 6 & ME, TE, LC & F 4, ML \\
\hline Sector 7 & IE, TE, TK & F 1 y F 4, GM y SM \\
\hline Sector 8 & ME, TE, TK & F 2 y F 4, ML y GW \\
\hline Sector 9 & ME, TE. & F 2 y F 4, ML y GW \\
\hline
\end{tabular}

Tabla 2. Característica de la coronación y talud en función de su estabilidad. El significado de las siglas y los sectores se indican en el texto. 


\section{Resultados}

\section{Condiciones ambientales que determinan la estabili- dad del terraplén}

Entre los factores que determinan la estabilidad del terreno en la zona con permafrost estudiada, se pueden señalar como los más significativos los siguientes: el régimen de temperatura estival, la hidrología superficial, la presencia de agua suprapermafrost, el desarrollo de la capa descongelamiento estacional o capa activa y las característica de las suelos sobre el techo del permafrost. A continuación se detallan las características de cada factor mencionado en el área de estudio. Uno de los problemas más graves que amenazan al $\mathrm{SMH} \mathrm{n}^{\circ} 38$, es la inestabilidad del terreno, compuesto por sedimentos y material de acarreo en la sección superficial durante el descongelamiento en el verano austral. Esto determina condiciones muy friables y poco compactadas que, sumadas al aumento en las últimas décadas del espesor de descongelamiento estacional, favorecen el desarrollo de los procesos de termokarst y termoerosión en el permafrost rico en hielo aumentando el asentamiento local de la estructura.

\section{Temperatura}

Se han evaluado las condiciones térmicas en el área del $\mathrm{SMH} \mathrm{n}^{\circ} 38$ a partir del análisis de los datos de la estación meteorológica más cercana, que es la de la Base Marambio. La información corresponde al periodo $1970-2010$ y procede del Servicio Meteorológico Nacional argentino. Las variaciones de la temperatura en este sector de la Península Antártica están directamente relacionadas con las condiciones anticiclónicas variables durante el año y con la acción moderadora del marde Weddell. Las temperaturas medias anuales oscilan entre los $-10^{\circ} \mathrm{C}$ y $-6,5^{\circ} \mathrm{C}$, las temperaturas medias de invierno son de alrededor de $-18^{\circ} \mathrm{C}$. Las temperaturas medias de verano entre $-3.5^{\circ} \mathrm{C}$ y $0.7^{\circ} \mathrm{C}$ (Figura 7 ).

Teniendo en cuenta el aumento de la temperatura producido por el calentamiento local en los sectores subpolares se pueden corregir los valores térmicos registrados hace 40 años para evaluar el régimen térmico actual (Silva Busso et al., 2009). Conforme a esta tendencia observada enel sector oriental de la Península Antártica y según los datos meteorológicos de la Estación Marambio la tendencia del aumento de la temperatura media anual en este sector es $0.05^{\circ} \mathrm{C} / \mathrm{año}$, y en el medio del verano es $0,06^{\circ} \mathrm{C} /$ año. También se debe corregir las temperaturas de

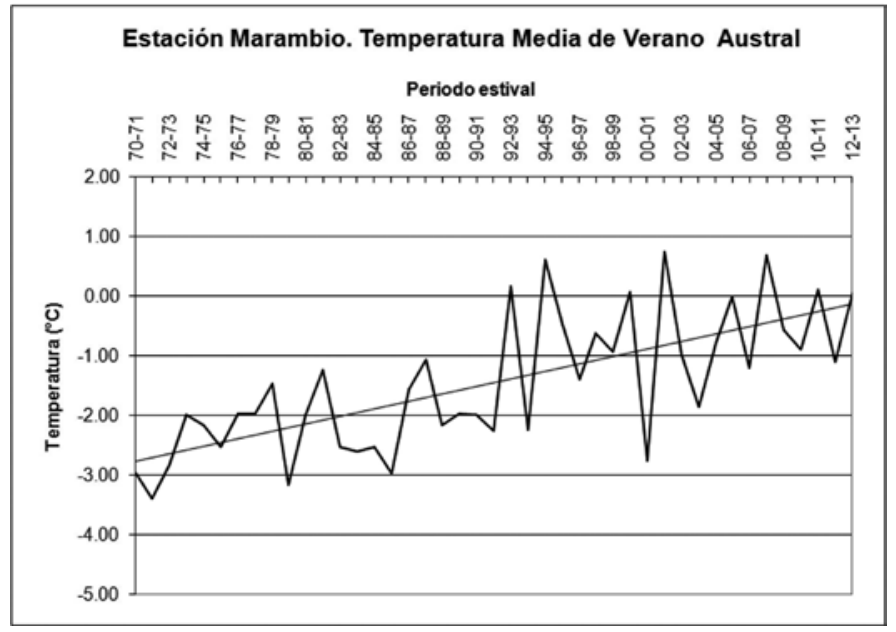

Figura 7. Temperatura media de verano durante 1970/2010 en la Base Marambio.

Figure 7. Summer mean temperature, $1970 / 2010$ in Marambio Station.

la Estación Marambio para referirlas al nivel del mar. Para ello se dispone de información tomada en la costa de la isla Marambio durante un año (dentro del periodo 2003-2005) que permite corregirla, obteniéndose un gradiente térmico de $0,7^{\circ} \mathrm{C} / 100 \mathrm{~m}$. Como resultado de la corrección propuesta la temperatura media anual en la zona costera es de $-6,0^{\circ} \mathrm{C}$ y la temperatura media del verano en la planicie de la isla Cerro Nevado es de $1,5^{\circ} \mathrm{C}$.

Con el objeto de determinar el espesor de la capa de descongelamiento estacional se procedió al cálculo del índice de grado-día positivo. El mismo se calcula a partir de la serie total de datos de temperatura, separando aquellos datos de temperatura diaria positiva y sumándolos entre sí, realizando previamente las correcciones antes mencionadas. El resultado del cálculo del índice de grado-día positivo corresponde avalores que podrían oscilar entre 300 y $600^{\circ} \mathrm{C} /$ día, que depende de las condiciones meteorológicas anuales.

\section{Hidrología superficial y formación de aguasuprapermafrost}

El sistema hídrico superficial está compuesto por múltiples vías de escorrentía de régimen temporal cuya actividad está fuertemente controlada por las condiciones meteorológicas, incluso durante los periodos estivales. El agua disponible en el sistema hídrico superficial se origina como consecuencia de la fusión de acumulacionesnivales usualmente abarlovento del terraplén. 
Sobre el terraplén las aguas superficiales pueden producir una erosión laminar relativamente uniforme de la superficie, debido sobre todo al movimiento del flujo sin que se formen cauces de agua de más de $0.30 \mathrm{~m}$ de profundidad. Sin embargo, la erosión laminar incluye numerosos surcos pequeños pero visibles causados por pequeñas concentraciones de escorrentía.

El régimen de las aguas suprapermafrost y el aporte del acuífero estival se relacionan con la fusión de la capa activa y las precipitaciones de verano, presentando caudal en los arroyos sólo en épocas estivales, según la porosidad de las rocas clásticas y las condiciones meteorológicas propias de cada verano en particular.

EI SEV realizado sobre el terraplén del SMH $n^{\circ}$ 38 (Figura 8) permite realizar una interpretación indirecta en función de las diferentes capas resistivas y su correlación con la geocriología e hidrogeología locales.

La capa más superficial, de $1.30 \mathrm{~m}$ de espesor, posee una resistividad de $30.9 \Omega \mathrm{m}$ que puede interpretarse como sedimentos areno-limosos con aguas dulces desarrolladas en la capa activa y con muy poca zona no saturada. La segunda capa resistiva infrayacente posee un espesor de $5.50 \mathrm{~m}$, llaga a una profundidad de 6.8 metros y presenta una resistividad mucho más elevada, de prácticamente 1960 $\Omega \mathrm{m}$. Este aumento de la resistividad es atribuiblea la presencia de hielo en zonas descongeladas con sedimentos arenosos finos y limos.

Finalmente, la tercera capa constituye la base de este SEV debido a queque no existe espacio para un tendido suficientemente largo para alcanzar mayor profundidad, pero arroja valores resistivos de $176 \Omega \mathrm{m}$ que como se ha señalado en trabajo previos (Fournier et al., 1990; Fukuda et al., 1992; Silva Busso et al., 2000, Silva Busso, 2009) se atribuye a la respuesta de permafrost seco (5-7\% de hielo) en sedimentos limo arcillosos, magnitud e interpretación típica de la zona de las islas Marambio y Cerro Nevado.

En general es un flujo de agua que, desde las zonas de saturación no permanente, circula a través de los estratos superiores a una velocidad alta y conformando una verdadera escorrentía subterránea. Debido a ello, los flujos de las aguas suprapermafrost provocan el desarrollo de los procesos de termo-abrasión del techo del permafrost.

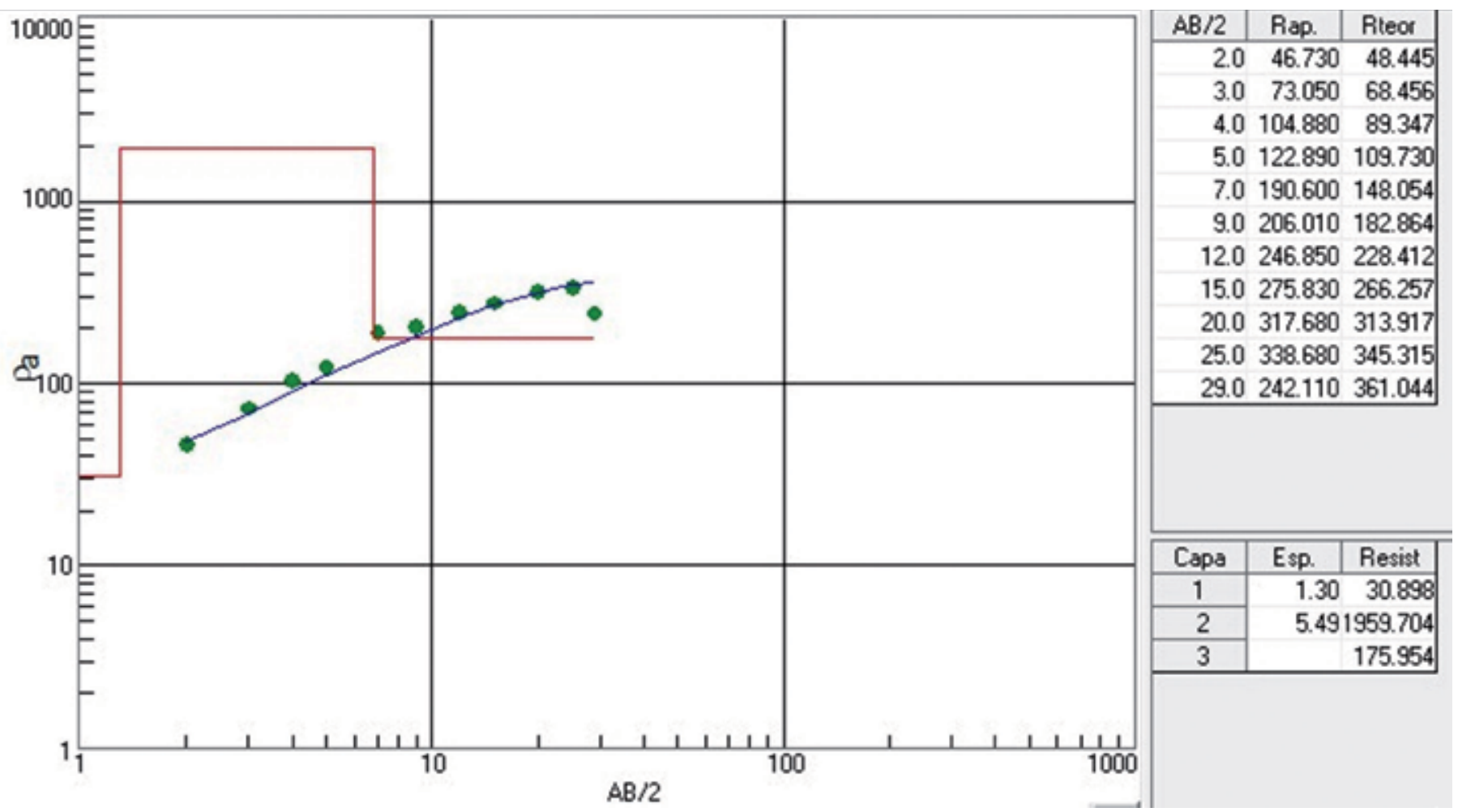

Figura 8. SEV realizado sobre el terraplén de la Cabaña deNordenskjöld. La segunda capa, de elevada resistividad, es interpretada como la respuesta de elevados contenidos de hielo.

Figure 8. VES on the top embankment of Nordenskjöld's Hut. The second layer, of high resistivity, is interpreted as the response of high ice content. 


\section{Condiciones geocriológicas y geotécnicas}

Originalmente la Cabaña de Nordenskjöld fue construida con la intención de servir como refugio en las condiciones ambientales antárticas por un período de aproximadamente un año. Fue traída de Suecia en forma prefabricada. El objetivo de la expedición no era el establecimiento de una base o refugio para una estancia más prolongada Por esta razón sus constructores no prestaron atención a las condiciones geotécnicas con perspectiva de conservarla a lo largo del tiempo. A consecuencia de ello el refugio se halla situado sobre un terraplén natural de la morena de fondo, con permafrost continuo rico en hielo. Esta ubicación determina condiciones geocriológicas y geotécnicas muy particulares y sensibles bajo las condiciones de cambio climáticode las últimas décadas (circunstancias imprevisibles al momento de la expedición sueca). El aumento de la temperatura estival, que se traduce en el aumento del índice grado día positivo, es el factor más importante, entre otros ya mencionados, que determina la inestabilidad.

Litológicamente los depósitos del terraplén en la parte superior se componen de fracciones clásticas finas, medianas y gruesas con contenido variable de arena fina - limo y relativamente poca arcilla. En secciones inferiores de la capa activa y el techo del permafrost se encuentran en general las fracciones más finas de arena media a fina con mayor contenido de limo y arcilla. En las capas superficiales del permafrost se hallan hielos intersticiales ocupando los espacios entre los sedimentos más gruesos, esto puede observares en la imagen de la Figura 9, tomada en la sección superior de la morrena de fondo.

\section{Discusión}

Los datos obtenidos en elcampo y laboratorio han permitido determinar el contenido en hielo subterráneo, que puede oscilar entre el $17-20 \%$, y en algunas secciones aisladas superar el $30 \%$. El espesor de la capa activa en la explanada del $\mathrm{SMH} \mathrm{n}^{\circ} 38$ alcanza 1.2 $\mathrm{m}$ en el flanco Sur, y este de 1,0 $\mathrm{m}$ en el flanco Este y $1.4 \mathrm{~m}$ en los flancos Norte y Oeste. Los hielos intersticiales en la morena del fondo local pueden observarse en el corte de sección de la figura 9.

Como se ha mencionado, el descongelamiento estacional es un factor muy variable, depende de la humedad de los suelos superficiales de la capa activa, el desarrollo de aguas suprapermafrost y la temperatura estival. La información básica mencionada ha sido empleada para evaluar el espesor local de la capa de descongelamiento usando la ecuación

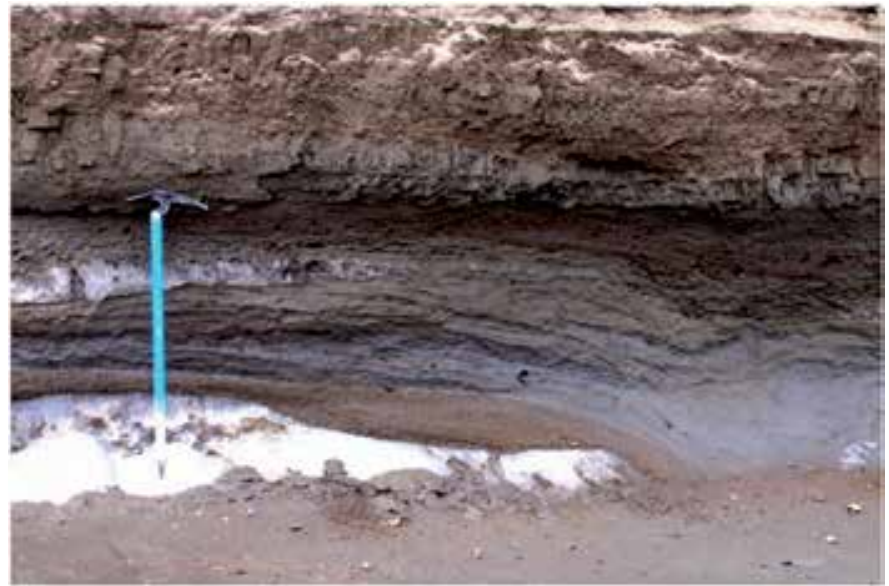

Figura 9. Techo del permafrost con hielo intersticial en la morrena del fondo.

Figure 9. Permafrost top with interstitial ice in hummocky moraine.

de Nelson derivada de la ecuación general de Stefan (Nelson et al., 1997). De acuerdo con dicho autor el espesor de descongelamiento $\left(Z_{d}\right)$ se calcula según. La ecuación (1).

$$
Z_{d}=\sqrt{\frac{156.4 n \lambda \Psi}{\rho W L}}
$$

Donde:

$n$ : Es el cociente entre la temperatura de la superficie del suelo y del aire, conocido como "factor- $n$ " (Shur and Slavin-Borovckiy, 1993);

$\lambda$ : Es la conductividad térmica del suelo descongelado

Y: Es el índice grado día positivo

$\rho$ : Es la densidad del suelo descongelado

$W$ : Es la relación de contenido total de agua por unidad de masa

\section{L: Es el calor latente de fusión del suelo congelado}

El factor $n$, calculado a partir de las series de temperatura corregidas y datos locales, es de -1.1, la conductividad térmica del suelo $(\lambda)$ fue medido en laboratorio en base a las muestras del terraplén y toma valores entre 1.6 y $1.7\left(\mathrm{Kcal} \mathrm{m}^{-1} \mathrm{~h}^{-1}{ }^{\circ} \mathrm{C}-1\right)$; el índice grado día positivo $(\Psi)$ se encuentra entre 300 y 600 $\left({ }^{\circ} \mathrm{Cd}\right)$, la densidad del suelo congelado $(\rho)$, también medida en laboratorio en las muestras del terraplén, toma valores promedio de $1.65\left(\mathrm{Tm}^{-3}\right)$, la relación de contenido total de agua por unidad de masa (W) se ha determinado en laboratorio entre 0.7 y 0.8 y finalmente el calor latente de fusión del suelo congelado 
(L) se ha tomado de tablas y para la litología mencionada se estima en 80000 (KcalTn ${ }^{-1}$ ). El resultado del cálculo, para toda la serie termométrica completa de la Estación Marambio determina que la tasa de $Z_{d}$ (espesor de descongelamiento) va a fluctuar entre 1.2 y $1.4 \mathrm{~m}$. No obstante, debido a que el original refugio fue establecido en 1902, cuando la temperatura media anual era al menos $2^{\circ} \mathrm{C}$ inferior a la actual (Nordenskjöld et al., 1907), por lo que se ha calculado el espesor de descongelamiento $Z_{d}$ considerando esta información inicial. De haberse mantenido estas condiciones el $Z_{d}$ previsto sería de entre $0.3-0.4 \mathrm{~m}$ por debajo del actual, lo que evidencia la influencia del cambio climático local en la zona de estudio. Durante los últimos cuarenta años esta tendencia al incremento de la temperatura y el consecuente aumento del espesor de descongelamiento estacional en este sector de la Península Antártica ha favorecido los procesos de asentamiento del terreno en toda la región pero con particular sensibilidad en las áreas de permafrost rico en hielo. El asentamiento potencial por aumento de descongelamiento estival se puede calcular mediante la expresión de la ecuación (2) propuesta por Shur and Slavin-Borovckiy (1993).

$$
A=\frac{\gamma_{e}\left(W_{t}-W_{l}\right)}{1+\gamma_{e}} h_{1}
$$

Donde:

A: Asentamiento potencial del terreno

$h_{l}$ : Espesor de la capa del suelo con hielos intersticiales afectado al aumento de descongelamiento estacional.

$W_{t}:$ Humedad total del suelo

$W_{i}:$ Es el contenido de humedad en el límite plástico del suelo

$\gamma_{e}:$ Es el peso específico del suelo seco.

El espesor de la capa del suelo con hielos intersticiales afectadopor el aumento de descongelamiento estacional $\left(h_{l}\right)$ es de $0.4 \mathrm{~m}$, la humedad del suelo $\left(W_{t}\right)$ fue medido en laboratorio en muestras del terraplén yalcanza valores entre $0.09-0.07$; el contenido de humedad en el límite plástico del suelo $\left(W_{i}\right)$ se encuentra en 0.25 y el peso específico del suelo seco $\left(\gamma_{\mathrm{e}}\right)$, también medida en laboratorio en muestras del terraplén, presenta valores promedio de $2.1\left(\mathrm{Tm}^{-3}\right)$. Con estos datos disponibles el resultado del asentamiento potencial $(A)$ en la explanada del terraplén estaría entre $0.15-0.25 \mathrm{~m}$. Este asentamiento potencial debe considerase como un valor máximo que sería potencialmente alcanzable los años de temperaturas más

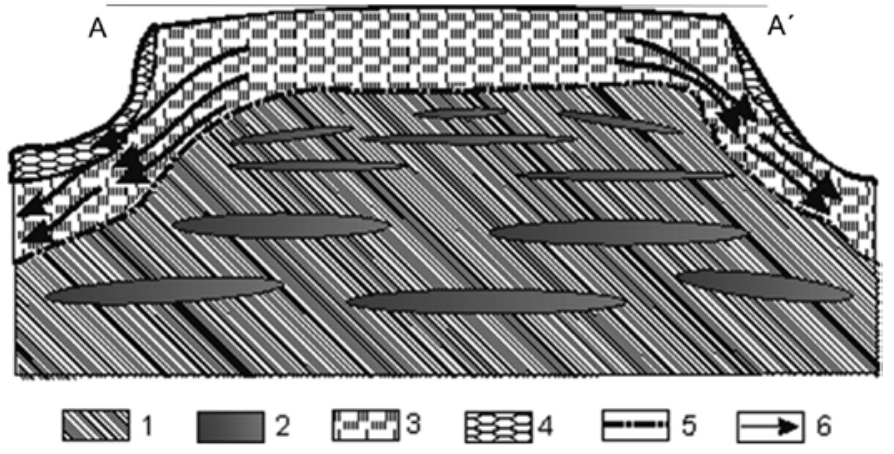

Figura 10. Esquema de la sección geocriogénica $A-A^{\prime}$ del terraplén. (ver situación en Figura 6). Pueden observarse: (1) zona de permafrost,(2) hielo subterráneo, (3) capa activa, (4) material usado como relleno artificial, (5) techo del permafrost y (6) movimiento del flujo de las aguas suprapermafrost.

Figure 10. Geocriogenic scheme section of embankment (A- $\left.A^{\prime}\right)$. (see location in Fig. 6). We can observe: (1) permafrost zone, (2) buried ice, (3) active layer, (4) material used as artificial filler, (5) permafrost top and (6) movement of the supra-permafrost groundwater flow.

altas, como el aquí analizado, pero en años de temperaturas medias más bajas (o más días con temperatura negativa) el valor de asentamiento se encontrará por debajo de esta cifra. La Figura 10 presenta un corte esquemático de la sección central del terraplén del $\mathrm{SMH} \mathrm{n}^{\circ} 38$. Este esquemamuestra la disposición de los aspectos geocriológicos actuales en el terraplén.

\section{Conclusiones}

Se concluye que el asentamiento del terraplén del $\mathrm{SMH} \mathrm{n}^{\circ} 38$ depende del contenido de hielo intersticial, del aumento del espesor de la capa de descongelamiento estacional producido por fusión del techo del permafrost y modificación de la superficie natural con daños significativos en la base del edificio. Se recomiendan acciones que eviten o al menos reduzcan la tasa de asentamiento, procurando la conservación de suelos a partir de la reducción de aquellas acciones que puedan alterar la coronación del terraplén. Como posible método de conservación se puede recomendar la utilizaciónde gaviones. Entre las principales funciones que desempeñarían se encuentran las de proteger a los márgenes y lechos de la erosión superficial y la termoerosión. También aumentarían la rugosidad en los márgenes del terraplén, mejorando la estabilidad de las pendientes. Como parte de las acciones de contingenciapara reducir los procesos de alteración de la coronación del terraplén que aumentarían su inestabilidad y colapso se proponen las siguientes tareas: 
1) Aumentar el espesor del terraplén en $0.25 \mathrm{~m}$ en la superficie de coronación con uso de áridos con una composición de arena con 30 a $40 \%$ de grava usando materiales locales con el propósito de reducir el asentamiento en este sector.

2) Realizar un armazón sobre la pendiente del terraplén con el empleo gaviones rellenos con materiales locales o transportados de otros sitios. El volumen calculado de áridos necesarios es no menor $65-70 \mathrm{~m}^{3}$.

3) En el talud del terraplén en los sectores 6, 7 y 8 que se encuentra sometido permanentemente a la erosión de las aguas superficiales durante el verano, se propone realizar un zócalo o barrera de contención con el objeto de retener el material y redistribuirlo nuevamente sobre la pendiente.

Estas tareas deberían realizarse bajo estricto control y consentimiento del personal arqueológico relacionado con la recuperación del edificio, dado que es muy frecuente el hallazgo de piezas de valor histórico en los alrededores del mismo. (RCTA, 2010, 2011). Las mencionadas tareas son coordinadas por el Proyecto de Conservación de Refugios Históricos (INST-07-2014, Área Museo, IAA).

\section{Referencias}

Capdevila, R. 1995. Informe de progreso del Programa Museo-Antar. Terceras Jornadas de Comunicaciones sobre Investigaciones Antárticas. Buenos Aires. p.449-450.

Comerci, S.M. 1983. Los trabajos de la República Argentina en la isla Cerro Nevado durante las campañas antártica 1979-1980 y 1980-1981.Contribución № 291 DNA, Buenos Aires. 21 p.

Ermolin, E. y Skvarca, P. 2004. Estudio de las variaciones de la capa activa en isla Marambio. $V^{\circ}$ Simposio Argentino y $I^{\circ}$ Latinoamericano sobre Investigaciones Antárticas. Buenos Aires, Resumen Expandido en CD-ROM, 4 p.

Fournier, H.G., Buk, E.M. y Corte, A.E. 1990. Three Permafrost Conditions Indicated by Geophysical Soundings in Tertiary Sediments at Seymour Island, Antarctic Peninsula. Cold Regions Science and Technology, 17, 301-307.

Fukuda, M., Strelin, J., Shimocawa, K., Takahashi, N., Sone, T. y Trombotto, D. 1992. Permafrost Occurrence of Seymour Island and James Ross Island, Antarctic Peninsula. In: Yoshida, E. (ed), Recent Progress in Antarctic Earth Science, , Tokyo, Japan p. 745-750.

Linell, K.A. y Jonston, G.H. 1973. Engineering design and construction in permafrost regions, 2nd. Int. Permafrost Conf., Yakutsk, North American Contr. Nat. Acad. Sci., 553-575.

Nelson F.E., Shiklomanov, N.I., Mueller, G., Hinkel, K.M., Walker, D.A. y Bockheim, J.G. 1997. Estimating active-layer thickness over a large region: Kuparuk River basin, Alaska, U.S.A. Arctic and Alpine Research, 29, 367-378.

Nordenskjöld, O., Andersson, J.G., Laesen, D.A. y Skottsberg, C. 1907. Viaje al Polo Sur: Expedición sueca a bordo del "Antarctik“, Vol- 1. Editorial Maucci, Buenos Aires, Argentina.

RCTA. 2003. Actividades Asociadas al Sitio y Monumento Histórico $N^{\circ}$ 38: Cabaña Cerro Nevado. XXVI Reunión Consultiva del Tratado Antártico Documento Informativo IP-057-AR Punto Agenda: VI CPA 4G, Argentina 3p.

RCTA. 2010. Actividades realizadas para la puesta en valor del SMH No 38 "Cerro Nevado". XXXIII Reunión Consultiva del Tratado Antártico, Documento Informativo IP21, Punta del Este, Uruguay. 4p.

RCTA. 2011. Actualización de las actividades realizadas para la puesta en valor del SMH N 38 "Cerro Nevado". XXXIV Reunión Consultiva del Tratado Antártico, Documento Informativo IP130, Buenos Aires, Argentina $4 p$.

Shur, Y.y Slavin-Borovskiy, V. 1993. N-factormaps of Russian permafrost region. Proceeding of the Sixth International Conference on Permafrost,Wushan Guangzhou, China, South China University of Technology Press, Vol. 1, 564-56.

Silva Busso, A. 2009. Aguas Superficiales y Subterráneas en el Área Norte de la Península Antártica. En: Silva Busso, A. (ed), El Agua en el Norte de la Península Antártica, Fundación Félix de Azara, Buenos Aires, Argentina, Cap. 4, 47-82.

Silva Busso, A., Fresina, M., Velasco, I. y Rey, C. 2009. La Península Antártica: Un Nuevo Horizonte para las Ciencias Hídricas. En: Silva Busso, A. (ed), El Agua en el Norte de la Península Antártica, Fundación Félix de Azara, Buenos Aires, Argentina, Cap.1, 1-12.

Silva Busso, A., Sánchez, R. y Fresina, M. 2000. Caracterización del Comportamiento Hidrogeológico en la Isla Marambio, Antártida. Primer Congreso Mundial Integrado de Aguas Subterráneas ALSHUD. $A / H$, Fortaleza, Brasil. Abstracs en CD-ROM, 292-307.

Recibido: julio 2013

Revisado: marzo 2014

Aceptado: octubre 2016

Publicado: marzo 2017 
